\title{
Instrumental characteristics of the Electron Spectrum Analyzer (ESA) onboard the Planet-B mission and observational perspectives of the electron measurements
}

\author{
S. Machida ${ }^{1}$, Y. Saito ${ }^{2}$, Y. Ito ${ }^{1}$, and H. Hayakawa ${ }^{2}$ \\ ${ }^{1}$ Department of Geophysics, Graduate School of Science, Kyoto University, Kyoto 606-8520, Japan \\ ${ }^{2}$ Institute of Space and Astronautical Science, Sagamihara, Kanagawa 229-0022, Japan
}

(Received September 26, 1997; Revised January 7, 1998; Accepted January 12, 1998)

\begin{abstract}
An Electron Spectrum Analyzer (ESA) onboard the Planet-B mission to the planet Mars will measure the electron energy flux in the range of $12 \mathrm{eV}$ to $15 \mathrm{keV}$ in 32 energy steps. The sensor is essentially a toroidal tophat electrostatic analyzer mounted on the surface of the spacecraft, which has a spin period of nominally 8 sec. With a $4^{\circ} \times 180^{\circ}$ view angle and its spinal motion, the sensor can measure three-dimensional electron velocity distribution functions. Such distribution functions will be taken mostly during the perigee path with a high bit rate burst mode. In a nominal low bit rate mode, the reduced distribution data in the pitch-angle and energy phase space will be recorded and transmitted to Earth together with the burst mode data.

This electron measurement will provide significant information for studies on the structure of the magnetosphere and ionosphere as well as information on particle acceleration and the wave-particle interaction processes around Mars.
\end{abstract}

\section{Introduction}

Since the first exploration by the Mariner 4 mission, more than 10 spacecraft have visited Mars and conducted measurements of the electromagnetic environment around the planet. However, little is known about the detailed signature of electrons and their roles such as in carrying the electric current, generating waves and interacting with the neutral atmosphere by collision in the Martian magnetosphere and ionosphere. The time resolution of the past electron measurements was too coarse to reveal useful information on the detailed structure of the Martian plasma environment.

Furthermore, observations and analysis of the three-dimensional electron velocity distribution functions have not been conducted yet. High-time resolution data are necessary to infer fine structures of the field aligned currents in the magnetosphere, and information on the velocity distribution functions is extremely useful in studying the particle acceleration processes, the wave-particle interaction processes, and collisional interaction with neutral particles in the Martian magnetosphere, as well as in the upper ionosphere. The Planet-B mission will be launched with an ISAS/M-V solid rocket, which imposes a relatively strict payload capacity restriction, i.e., approximately $33 \mathrm{~kg}$, so that is necessary to minimize its size and weight of each payload to enhance efficiency and to maximize the total scientific outcome of this mission.

\section{Instrumentation}

The Electron Spectrum Analyzer (ESA) was designed to measure the thermal and hot electrons in the Martian magnetosphere and upper ionosphere. The sensor is basically a

Copy right (C) The Society of Geomagnetism and Earth, Planetary and Space Sciences (SGEPSS); The Seismological Society of Japan; The Volcanological Society of Japan; The Geodetic Society of Japan; The Japanese Society for Planetary Sciences. toroidal top-hat electrostatic analyzer, as shown in Figs. 1 and 2. This type of sensor has the advantage that it realizes a large geometrical factor with a small-size and light-weight configuration, which is the principle concept to maximize the capability of the Planet-B mission with a limited payload capacity, as explained earlier. The diameter of the top-hat plate is $90 \mathrm{~mm}$, and the radius of the toroidal electrode center is $30 \mathrm{~mm}$ with a $5 \mathrm{~mm}$ offset from the center of the top-hat plate. The separation between the inner and outer electrodes is $3.0 \mathrm{~mm}$, as shown in Fig. 1. (realizing a geometrical factor of $2.0 \times 10^{-5}(\mathrm{eV} / \mathrm{eV}) \mathrm{str} \mathrm{cm}^{2}$ for $\left.4^{\circ} \times 5.6^{\circ}\right)$ The optimum design of the sensor was determined by conducting numerical particle ray tracing calculations.

The view angle of the ESA is $4^{\circ}$ in its full width in the azimuthal direction and $180^{\circ}$ in the elevational direction with respect to the spacecraft spin axis. In principal, the latter can be taken to be $360^{\circ}$ for the top-hat analyzer. However, due to interference of other instruments, the view angle of the ESA was set to $180^{\circ}$. The angular resolution is $4^{\circ}$ in the azimuthal direction and $5.6^{\circ}$ in the elevational direction. The ESA is placed so that its rotational symmetric axis is perpendicular to the spacecraft spin axis.

The energy sweep of the measured electrons is performed by varying the applied voltage to the inner toroidal electrode, while keeping the voltage of the outer electrode at the ground level. There are four memory areas to store the energy tables controling the sweeping voltage unit which can produce an output from 0 to $2000 \mathrm{~V}$ with the use of a high voltage diode unit (HV601) to which a regulated high voltage of $3000 \mathrm{~V}$ is supplied externally. The measurable energy range of the electrons is from $12 \mathrm{eV}$ up to $15 \mathrm{keV}$. The energy tables prepared are at present High Energy mode, Logarithmic/Hot mode, Linear/Hot mode, and Low Energy mode, and the energy scan will be performed with a logarith- 


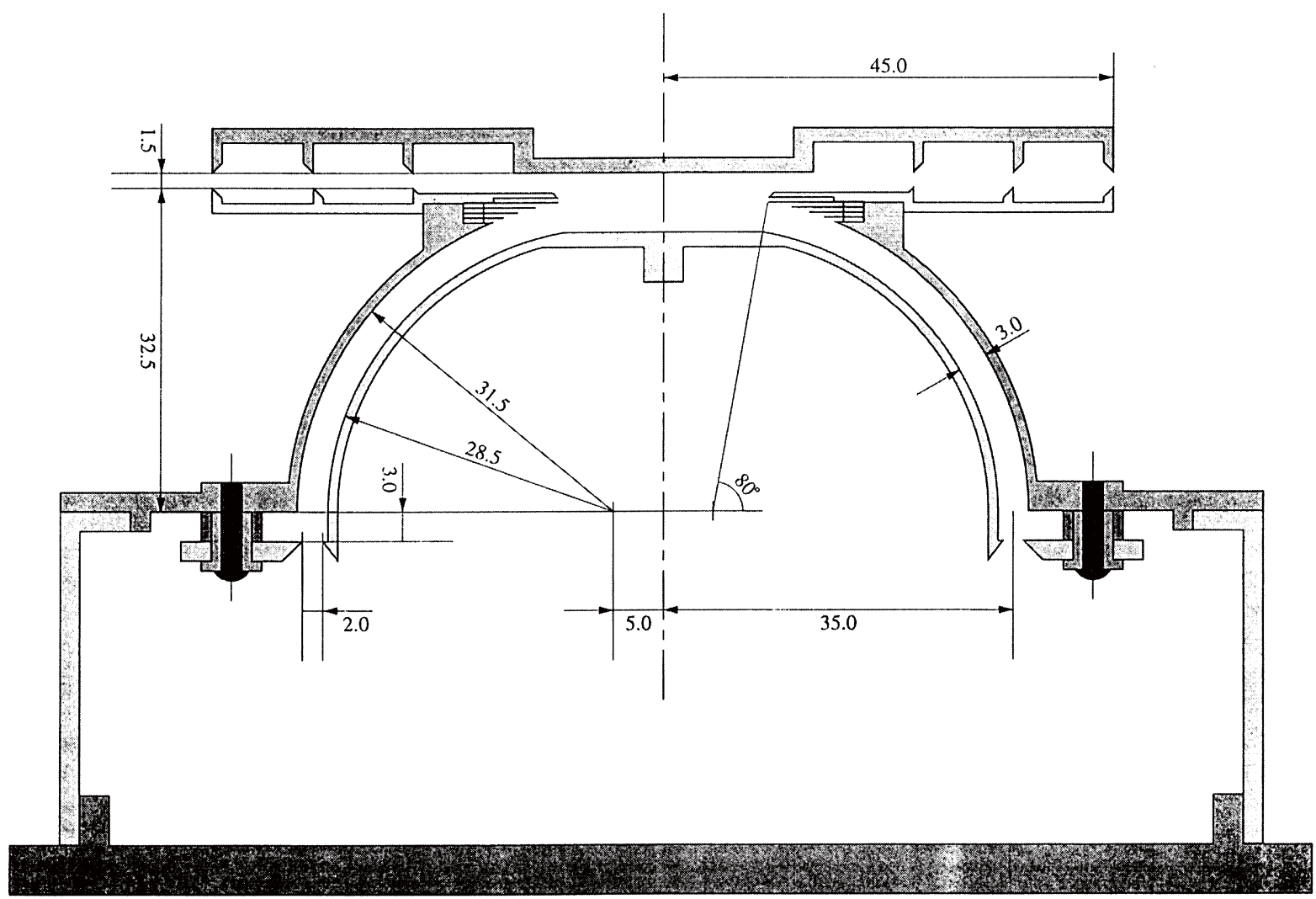

Fig. 1. The design of the ESA which has the architecture of a top-hat electrostatic analyzer.

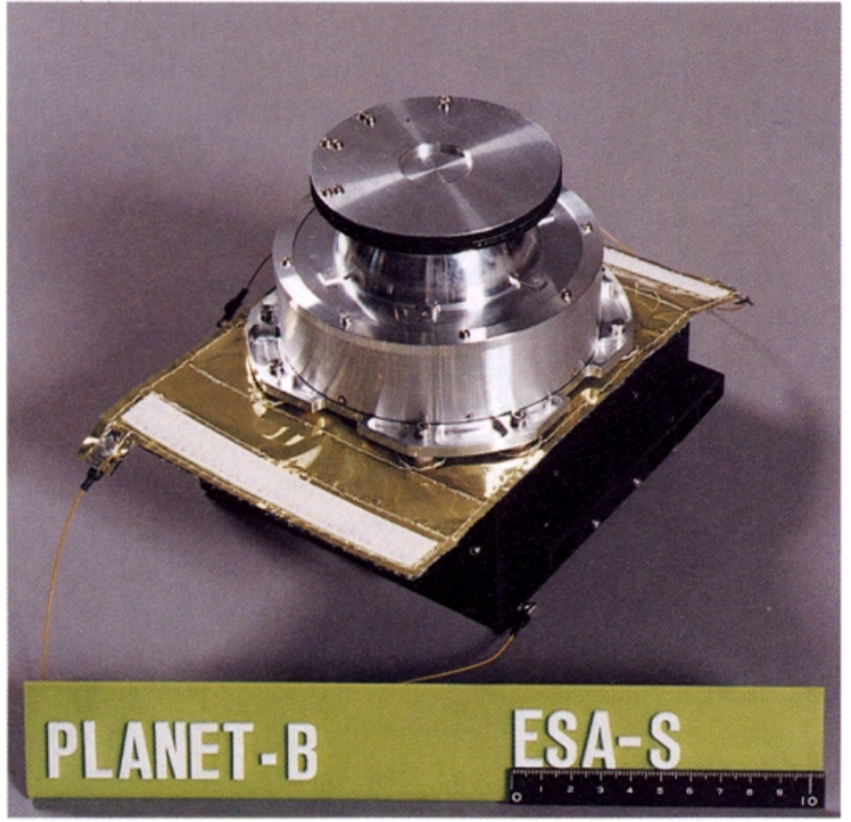

Fig. 2. Photograph of the ESA.

mically equal width in the former two modes, and a linearly equal width in the latter two modes.

The electrons transmitted through the electrodes are received by a three-stage micro-channel plate (MCP) of annular shape, and intensified to detectable charge pulses. A negative potential mesh $(-12 \mathrm{~V})$ is placed between the exit of the electrodes and the MCP so as to prevent the entrance of secondary electrons and to suppress spurious counts. The charge pulses are received by a position-sensitive resistive anode. The position of the charge pulse indicates the elevational angle at the time of entrance for an incoming electron.

With its $8 \mathrm{sec}$ spin rotation, the ESA can measure the whole three-dimensional energy spectrum, i.e., the velocity distribution function with that time resolution. There are various data acquisition rates, between $64 \mathrm{bps}$ and $64 \mathrm{kbps}$, available for the Planet-B mission. Direct three-dimensional velocity distribution functions will be stored in a data recorder for approximately $20 \mathrm{~min}$ at a recording rates of 64 $\mathrm{kbps}$ or 40 min at $32 \mathrm{kbps}$. This high bit rate burst mode will be mostly conducted in a perigee mode to survey the detailed structures of the upper atmosphere and its interaction with the solar wind. The orbital velocity of the spacecraft is typically $5 \mathrm{~km} / \mathrm{s}$ around the perigee, hence, the spatial resolution with this mode is $\sim 40 \mathrm{~km}$, which is enough to infer the structure of the shock and upper atmosphere of Mars. In a nominal mode, the recording rate will be basically 64,128 or $256 \mathrm{bps}$. When the recording rate is below $8 \mathrm{kbps}$, the measured distribution functions of the electrons are sorted with respect to the local magnetic field provided by an onboard magnetic field sensor (MGF). The typical time resolution of the low bit rate electron data is $128 \mathrm{sec}$ with a 128 bps transmission rate. Furthermore, the pressure tensor of electrons is calculated optionally with an onboard microprocessor (H32OBC) with a typical time resolution of $128 \mathrm{sec}$. Together, this and the fact that the orbital velocity 
Table 1. Summary of the instrumental specifications of the ESA.

\begin{tabular}{ll}
\hline Energy range & $12 \mathrm{eV} \sim 15 \mathrm{keV}$ \\
Energy step & $32 \mathrm{steps}$ \\
Energy resolution & $\Delta E / E \sim 7 \%$ \\
Field of view (Intrinsic) & $4^{\circ} \times 180^{\circ}$ \\
Angular resolution & $4^{\circ} \times 5.6^{\circ}$ \\
G-factor & $2.0 \times 10^{-5} \mathrm{~cm}^{2} \mathrm{str} \mathrm{eV} / \mathrm{eV}$ for $4^{\circ} \times 5.6^{\circ}$ \\
Data acquisition mode & $3 \mathrm{D}$ velocity distribution function (when the bit rate is equal to or higher than $8 \mathrm{kbps})$ \\
& 2D distribution function sorted by pitch angle (when the bit rate is lower than $8 \mathrm{kbps})$ \\
Typical time resolution & 3D mode: $8 \mathrm{sec}($ at $64 \mathrm{kbps})$ \\
& 2D mode: $128 \mathrm{sec}($ at $128 \mathrm{bps})$ \\
\hline
\end{tabular}

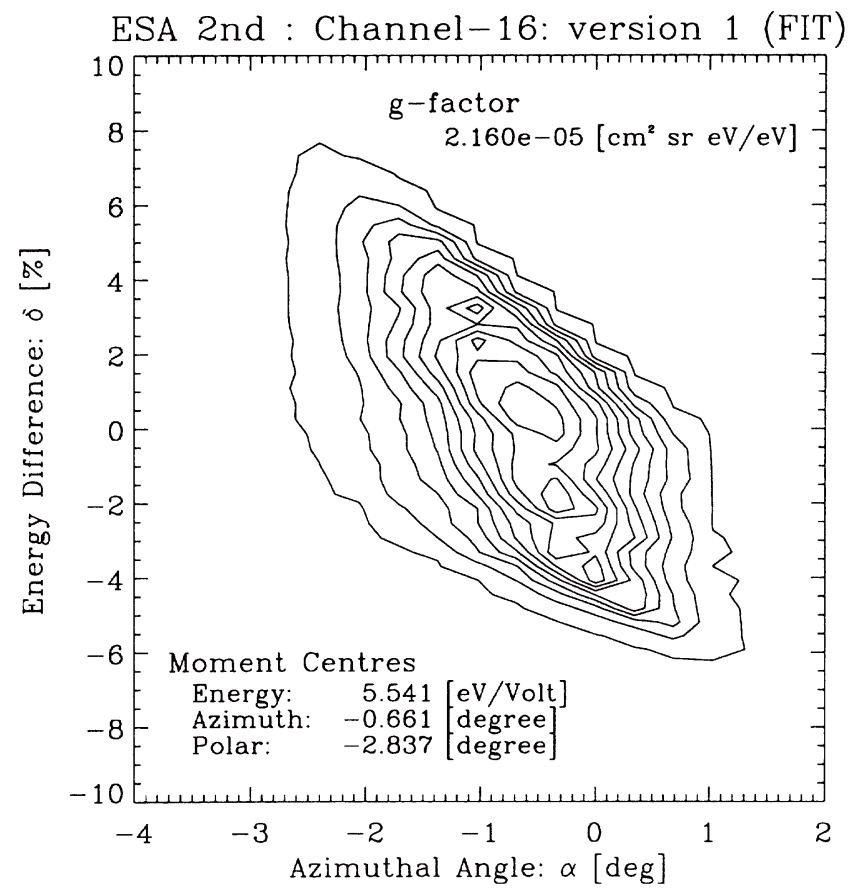

(a)

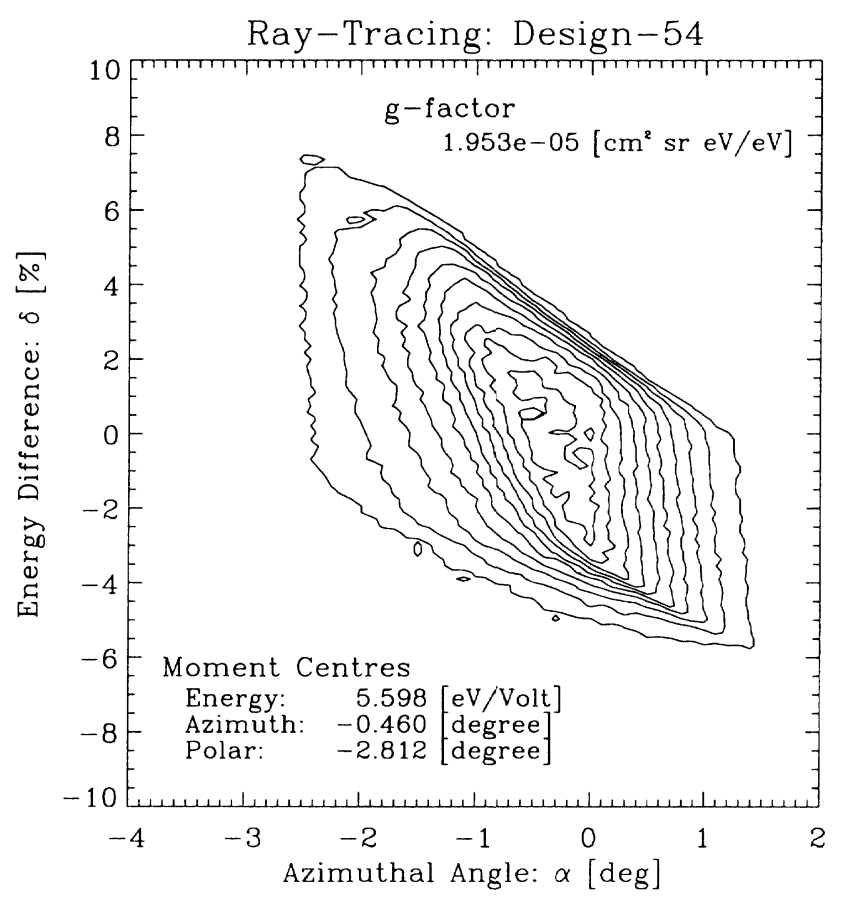

(b)

Fig. 3. Instrumental response of the ESA displayed on the plane of the azimuthal angle-particle energy. (a) Experimental result (b) Theoretical prediction.

of the spacecraft at apogee is $\sim 350 \mathrm{~m} / \mathrm{s}$, yield a spatial resolution of $45 \mathrm{~km}$, which is quite satisfactory to infer the structure of the plasma environment or of the wave-particle interaction processes around Mars. The 12 bit particle count data is compressed into 8 bit data by the method employed in the previous sensor onboard the GEOTAIL spacecraft (Mukai et al., 1994). In the Planet-B mission, we will further reduce the data length by employing an "arithmetic cording", a data compression method selected after comparison with other methods applied them to the terrestrial data from the GEOTAIL spacecraft. Those parameters are summarized in Table 1.

The sequence of the high voltage sweep, data compression and data handling are all performed by an H32OBC microprocessor placed in an electronic PSA (Particle Spectrum Analyzer) unit. The PSA has another microprocessor (80C86)and it controls not only the ESA but also an ion sensor (ISA), a high energy particle sensor (EIS), and an extreme ultraviolet imager (XUV), simultaneously. Such integration could reduce the total weight of the instruments significantly. A detailed description of the PSA is given in a paper by Hayakawa et al. (1998).

The flight module of the ESA was fabricated and its performance tested with the calibration facility of the low energy particle instruments at ISAS. The calibration was performed with a singly charged nitrogen molecular ion beam with an energy of $8 \mathrm{keV}$ by applying the negative potential to the inner toroidal electrode. A number of electron sensors have been calibrated with this method in the past, and the legitimacy of the technique has been well established. Figure 3(a) shows an example of the experimental results showing the instrumental response in a plane of particle energy and azimuthal angle. This has to be compared with Fig. 3(b) in which a theoretical prediction, i.e., the result of the numerical particle ray tracing, is shown. The agreement between the two results is quite satisfactory. A detailed description of the sensor calibration and final characteristics will be given elsewhere. 


\section{Perspectives of the Electron Measurement} around the Planet Mars

Within an MHD regime, it is known that electrons are carriers of electric current along the magnetic field as well as being carriers of perpendicular current. The ions are mostly carriers of perpendicular current. When the scale length of a specific region is smaller than the ion Larmor radius of some species, these ions can be regarded as unmagnetized. On the contrary, we can assume that the electrons are magnetized in most cases. For example, the magnetic field around Mars is so weak, typically $20-30 \mathrm{nT}$, that the Larmor radius of the $400 \mathrm{~km} / \mathrm{s}$ pick-up singlycharged oxygen ion is $3400-2200 \mathrm{~km}$, which is comparable to the radius of the planet. Therefore, the finite radius effect of those ions is significant in the bow shock or the planetopause region. The spatial distribution of the unmagnetized ions is probably diffusive and gradual, but the distribution of the electrons are likely to contain smaller structures, since they are strongly magnetized. The electrons would show a sharp transition such as in the number density, temperature or velocity distribution functions. Those boundary regions may be easily detected by electron measurements. The roles of electrons and ions are different, and an understanding these differences obtained by comparing these with those for Earth would be extremely important.

In the bow shock region, there would be an electron thermal heating process similar to that around Earth or other planets (Feldman et al., 1983; Shimazu et al., 1996). Electron plasma oscillation has been observed in the foreshock region of the planet Mars (Grard et al., 1989). This can be excited by the beam interaction between the solar wind electrons and the back-streaming electrons reflected at the Martian bow shock. The study of the electron signature around the shock is important in constructing the framework for the theory of the shock, nonlinear plasma dynamics, and the particle acceleration process.

The structure of the planetopause is highly dependent on the answer to the question of whether the planet Mars has an intrinsic magnetic field or not. Recent observations of the Mars Global Surveyor indicates that the surface magnetic field of Mars is less than $40 \mathrm{nT}$. Therefore, the interaction between the solar wind and Mars is akin to the Venus case, i.e., the solar wind dynamic pressure is balanced by the thermal pressure of the Martian upper atmosphere. At the same time, the Mars Global Surveyor discovered very localized magnetic anomalies for which the onboard magnetometer indicated values of about $400 \mathrm{nT}$ below the ionospheric altitude (Showstack, 1997). We can expect very localized anomalies of plasma transport such as we observe in a cusp region of Earth, where a characteristic structure with a notable energy dispersion can be found in the particle energy-time diagram (e.g., Lin et al., 1986). As mentioned earlier, electrons are strongly magnetized and suitable for detecting sharp boundaries or small structures, therefore, such a signature can be easily found in electron data. Also, close inspection of the loss cone in the electron velocity distribution would tell us the ratio of the magnetic field intensity at the spacecraft position and that of the foot point of the magnetic field line traced down to the planetary ionosphere. It is possible to detect a localized magnetic anomalies of Mars at the ionospheric altitude using this method (Howe et al., 1974).

A major source of thermal electrons is the photo-ionization in the dayside ionosphere of Mars. The primary photoelectrons have energies of between 20 and $30 \mathrm{eV}$, which may create several peaks in that energy range of the electron spectrum. There will be ample chemical reactions between the neutral particles and electrons, which determine the final structure of the electron spectrum. A detailed measurement of the electron energy spectrum will provide extremely valuable data in discussing the structure and chemical processes in this region. Additionally, we can determine the spacecraft charging potential by comparing the energy shift of the observed electron spectrum in the Martian ionosphere with the expected one, once we establish an understanding of the relevant structure of the ionospheric electrons. Also, the same technique can be applied to artificial photoelectrons from the spacecraft surface to deduce the spacecraft charging potential.

As for the night-side ionosphere, it is known that the peak density of plasma is often below $10^{3} \mathrm{~cm}^{-3}$ which is the threshold of the radio occultation measurement and already about 200 times smaller than that of the day-side ionosphere (Zhang et al., 1990). Observations on the night-side ionosphere of Mars have been carried out only by occultation measurements, and no direct observations have been conducted so far. In the night-side ionosphere of the planet Venus, it is know that the plasma density is relatively high, and there are several distinct structures such as an ionospheric hole, a ray, and a flux rope (Brace et al., 1987). The question of whether all of these structures exist in the Martian nightside ionosphere or not is of interest in terms of a comparative study of the planetary upper ionosphere. Such a study can be conducted by investigating the data obtained by direct measurement of the electrons together with the data of ions, magnetic fields and waves from the Planet-B mission. In particular, the electrons can be strongly magnetized and carry the current in those regions. Therefore, our measurements will be one of the key observations in studying on the night-side ionosphere of Mars.

The night-side tail of Mars is characterized by oxygen ion beams $\left(\mathrm{O}^{+}, \mathrm{O}^{++}\right)$and several other ions such as $\mathrm{CO}_{2}{ }^{+}$which are regarded to originate from the Martian upper atmosphere (Lundin et al., 1989). A notable feature of these ion beams is that their kinetic flow energies are the same regardless of the mass per charge ratio, which implies that the acceleration is due to an electrostatic potential drop such as found in the auroral region of Earth. From this fact, Lundin et al. (1989), who first reported this component, called them the "auroral ion beams". A recent paper by Dubinin et al. (1993) proposes a model to explain such an acceleration due to the electrostatic potential structure in which the ions are unmagnetized but the electrons are strongly magnetized. If we admit the frozen-in relation for the electrons, the existence of the electrostatic field, i.e., an electrostatic potential drop is required in the night-side near the tail region of Mars. However, if such an electrostatic field exists, there may also be some electron acceleration which appears as an inertia term neglected in their paper. Also, the pressure gradient term which was neglected in their study, should be quanti- 
tatively investigated by observation.

Information on the pitch angle distribution of the electrons is useful for tracing the magnetic field line. For example, in the plasma vortices in the low latitude boundary layer (LLBL) of Earth, field aligned bi-streaming electron beams are commonly observed. The field line characterized by this bi-streaming electron beam is regarded as being a closed magnetic field, both ends of which are connected to the planet (Ogilvie et al., 1984). We can apply the same method to Mars to determine whether there are actually closed field lines or not, and if so, how they extend away from the planet.

\section{Conclusions}

The electron spectrum analyzer ESA is composed of a toroidal top-hat electrode, an annular MCP with a position sensitive anode, charge amplifiers, and high voltage supply units. The performance of the ESA has been tested using the calibration facility of the low energy particle sensor at ISAS. The experimental results agree well with the theoretically calculated results, e.g., in the form of an energy-angle characteristic diagram. There are several key regions in understanding the structure of the plasma environment around Mars, i.e., bow shock, planetopause, day-side ionosphere, night-side ionosphere, and night-side tail regions. Above the region of the locally enhanced surface magnetic field, the dynamic pressure of the solar wind balances with the magnetic pressure and some anomalies can be detected by the electron measurements. The data from the electron measurements will provide significant information on the electromagnetic plasma environment around the planet Mars.

Acknowledgments. The authors would like to thank T. Mukai and other coinvestigators of the ESA team for their valuable suggestions and comments. The sensor was fabricated by the Mitaka-Koki Co., and the microchannel plate and the position sensitive anode were manufactured by the Hamamatsu-PhotoElectronic Co. The electronic units were all manufactured by the Meisei-Electronic Co.

\section{References}

Brace, L. H., W. T. Kasprzak, H. A. Taylor, R. F. Theis, C. T. Russel, A. Barnes, J. D. Mihalov, and D. M. Hunten, The ionotail of Venus: its configuration and evidence for ion escape, J. Geophys. Res., 92, 15-26, 1987.

Dubinin, E., R. Lundin, O. Norberg, and N. Pissarenko, Ion acceleration in the Martian tail: Phobos observations, J. Geophys. Res., 98, 3,9913,997, 1993.

Feldman, W. C., R. C. Anderson, S. J. Bame, S. P. Gary, J. T. Gosling, D. J. McComas, M. F. Thomsen, G. Paschmann, and M. M. Hoppe, Electron velocity distributions near the earth's bow shock, J. Geophys. Res., 88, 96-110, 1983.

Grard, R., A. Pedersen, S. Klimov, S. Savin, A. Skalsky, J. G. Trotignon, and C. Kennel, First measurements of plasma waves near Mars, Nature, 341, 607-609, 1989.

Hayakawa, H., Y. Saito, and Y. Ito, Instrumental characteristics of the ion spectrum analyzer (ISA) onboard the Planet-B mission and observational perspectives of the ion measurements, in preparation, 1998.

Howe, H. C., R. P. Lin, R. E. McGuire, and K. A. Anderson, Energetic electron scattering from the lunar remanent magnetic field, Geophys. Res. Lett., 1, 101-104, 1974.

Lin, C. S., J. L. Burch, and J. D. Winningham, Near-conjugate observations of polar cusp electron precipitation using DE-1 and DE-2, J. Geophys. Res., 91, 11,186-11,202, 1986.

Lundin, R., A. Zakharov, R. Pellinen, H. Borg, B. Hultqvist, N. Pissarenko, E. M. Dubinin, S. W. Barabash, I. Liede, and H. Koskinen, First measurements of the ionospheric plasma escape from Mars, Nature, 341, 609-612, 1989.

Mukai, T., S. Machida, Y. Saito, M. Hirahara, T. Terasawa, N. Kaya, T. Obara, M. Ejiri, and A. Nishida, The low energy particle (LEP) experiment onboard the GEOTAIL satellite, J. Geomag. Geoelectr., 46, 669-692, 1994.

Ogilvie, K. W., R. J. Fitzenreiter, and J. D. Scudder, Observations of electron beams in the low-latitude boundary layer, J. Geophys. Res., 89, 10,723-10,732, 1984.

Shimazu, H., S. Machida, and M. Tanaka, Macroparticle simulation of collisionless parallel shocks generated by solar wind and planetary plasma interactions, J. Geophys. Res., 101, 7,647-7,658, 1996.

Showstack, R., Mars has crustal, complex magnetic field, EOS Trans. AGU, 78, 429, 1997.

Zhang, M. G. H., J. G. Luhmann, and A. J. Kliore, An observational study of the nightside ionospheres of Mars and Venus with radio occultation methods, J. Geophys. Res., 95, 17,095-17,102, 1990.

S. Machida (e-mail: machida@kugi.kyoto-u.ac.jp), Y. Saito, Y. Ito, and H. Hayakawa 